Yury A. Rossikhin* and Marina V. Shitikova*

\title{
Analysis of damped vibrations of thin bodies embedded into a fractional derivative viscoelastic medium
}

\begin{abstract}
Damped vibrations of elastic thin bodies, such as plates and circular cylindrical shells, embedded into a viscoelastic medium, the rheological features of which are described by fractional derivatives, are considered in the present article. Besides the forces of viscous friction, a thin body is subjected to the action of external forces dependent on the coordinates of the middle surface and time. The boundary conditions are proposed in such a way that the governing equations allow the Navier-type solution. The Laplace integral transform method and the method of expansion of all functions entering into the set of governing equations in terms of the eigenfunctions of the given problem are used as the methods of solution. It is shown that as a result of such a procedure, the systems of equations in the generalized coordinates could be reduced to infinite sets of uncoupled equations, each of which describes damped vibrations of a mechanical oscillator based on the fractional derivative Kelvin-Voigt model.
\end{abstract}

Keywords: cylindrical shell; damped vibrations; fractional derivative viscoelasticity; fractional oscillator; thin plate.

*Corresponding authors: Yury A. Rossikhin and Marina V. Shitikova, Research Center for Wave Dynamics in Solids and Structures, Voronezh State University of Architecture and Civil Engineering, 20-letijya Oktjabrja Street 84, Voronezh 394018, Russia, e-mail:YAR@vgasu.vrn.ru; shitikova@vmail.ru

\section{Introduction}

The interest in fractional calculus has increased profoundly in the past few decades, resulting in a large body of articles devoted to this challenge, which is clearly emphasized in a set of review articles published in the field [1-6]. The most recent state-of-the-art article [6] is devoted to the analysis of new trends and recent results during the last decade, in the field of fractional calculus application to the mechanics of materials and the dynamic problems of structural mechanics, while a historical survey about two parallel ways in the progress of fractional calculus in the mechanics of solids can be found by the interested reader in ref. [7].

The review by Rossikhin and Shitikova [6] has shown that during the last decade, fractional calculus entered the mainstream of engineering analysis and has been widely applied to structural dynamics problems both in discrete and continuous equations. However, the majority of articles are mainly concerned with the investigation of vibrations of one-degree-of-freedom systems.

In the present article, it will be shown how, using the Raleigh hypothesis and the modal analysis, the dynamic problems of the systems with an infinite number of degrees of freedom, such as thin plates and shells, can be reduced to a finite or an infinite set of uncoupled linear oscillators, the theory of which is well developed.

\section{Damped vibrations of $\mathrm{N}$-degree- of-freedom mechanical systems}

It is known [8] that during the analysis of linear damped vibrations of $\mathrm{N}$-degree-of-freedom mechanical systems, their equations of motion could be reduced to a finite set of uncoupled equations in the generalized coordinates, each of which describes damped vibrations of a mechanical oscillator. Really, the equation of motion of such systems in the vector form could be written as

$$
m \overrightarrow{\vec{V}}+c \overrightarrow{\dot{V}}+k \vec{V}=\vec{P}(t),
$$

where $\vec{V}$ is the displacement vector, an overdot denotes the derivative with respect to the time $t, m$ is the matrix of lumped masses with the elements $m_{n}, k$ is the stiffness matrix with the elements $k_{n}, c$ is the damping matrix with the elements $c_{n}$, and $\vec{P}(t)$ is the force vector. Suppose that

$$
c=a_{0} m+a_{1} k
$$

and represent the solution of Eq. (1) and the force $P(t)$ in terms of the expansions in eigenvectors $\varphi_{i}(i=1, \ldots, N)$ of the given problem, i.e., 


$$
\begin{aligned}
& V=\varphi_{1} Y_{1}+\varphi_{2} Y_{2}+\ldots+\varphi_{N} Y_{N}, \\
& P(t)=\varphi_{1} P_{1}+\varphi_{2} P_{2}+\ldots+\varphi_{N} P_{N},
\end{aligned}
$$

then after the substitution of Eq. (3) in Eq. (1) with accounting for the orthogonality conditions of the eigenvectors

$\varphi_{m}^{\mathrm{T}} m \varphi_{n}=\varphi_{m}^{\mathrm{T}} k \varphi_{n}=\varphi_{m}^{\mathrm{T}} c \varphi_{n}=0 \quad(m \neq n)$,

$\varphi_{m}^{\mathrm{T}} m \varphi_{n}=M_{n}, \quad \varphi_{m}^{\mathrm{T}} k \varphi_{n}=\omega_{n}^{2} M_{n}, \quad \varphi_{m}^{\mathrm{T}} c \varphi_{n}=\tau_{n} \omega_{n}^{2} M_{n} \quad(m=n)$,

and after applying the Laplace transformation, we are led to the following equations:

$$
\left(p^{2}+\omega_{n}^{2} \tau_{n} p+\omega_{n}^{2}\right) \bar{Y}_{n}=\bar{F}_{n} \quad(i=1, \ldots, N),
$$

where $p$ is the Laplace transform parameter; $\bar{F}_{n}=\bar{P}_{n} M_{n}^{-1}$, and an overbar denotes the Laplace transform; $a_{0}$ and $a_{1}$ are the coefficients of proportionality; $Y_{i}$ and $P_{i}(i=1, \ldots, N)$ are the generalized displacements and forces, respectively; an upper index T denotes the transposed vector; $\omega_{n}$ is the $n$-th frequency of natural vibrations; and $\tau_{n}$ is the retardation time for the $n$-th mode.

Each equation from Eq. (5) describes the damped vibrations of a mechanical oscillator, the damping features of which are described by the classic Kelvin-Voigt model.

If the Kelvin-Voigt model involves a fractional derivative, then Eq. (5) is written as [1, 9]

$$
\left(p^{2}+\omega_{n}^{2} \tau_{n}^{\gamma} p+\omega_{n}^{2}\right) \bar{Y}_{n}=\bar{F}_{n} \quad(i=1, \ldots, N),
$$

where $\gamma(0<\gamma \leq 1)$ is the order of the fractional derivative (fractional parameter).

Reference to Eqs. (5) and (6) shows that the viscosity of the mechanical system has a modal character, which is verified by the experimental data [10].

From Eq. (6), we find

$$
\begin{gathered}
\bar{Y}_{n}=\frac{\bar{F}_{n}}{\bar{f}_{n}} \quad(i=1, \ldots, N), \\
\bar{f}_{n}=p^{2}+\omega_{n}^{2} \tau_{n}^{\gamma} p+\omega_{n}^{2} .
\end{gathered}
$$

To convert from the Laplace domain to the time domain through the Mellin-Fourier inversion formula

$$
Y_{n}(t)=\frac{1}{2 \pi i} \int_{c-i \infty}^{c+i \infty} \bar{Y}_{n}(p) \mathrm{e}^{p t} \mathrm{~d} p,
$$

all particular points of the function $\bar{Y}_{n}(p)$ need to be found. This function possesses the branch points $p=0$ and $p=-\infty$ and has the simple poles at the same magnitudes of $p$, which are the roots of the characteristic equation

$$
\bar{f}_{n}=0 \text {. }
$$

As for the multivalued functions possessing branch points, the inversion formula is valid only for the first sheet of the Riemann surface; then, for calculating the integral in Eq. (9), the closed contour of integration with a cut along the real negative axes should be used [1,9]. Applying Jordan lemma and the main theorem of the residue theory, the integral in Eq. (9) can be written in the form

$$
\begin{gathered}
Y_{n}(t)=Y_{n}^{\text {drift }}(t)+Y_{n}^{\text {vibr }}(t), \\
Y_{n}^{\text {drift }}(t)=\frac{1}{2 \pi i} \int_{0}^{\infty}\left[\bar{Y}_{n}^{(}\left(s e^{i \pi}\right)-\bar{Y}_{n}\left(s e^{i \pi}\right)\right] e^{s t} \mathrm{~d} s, \\
Y_{n}^{\text {vibr }}(t)=\sum_{k} r e s\left[\bar{Y}_{n}\left(p_{k}\right) e^{p_{k} t}\right],
\end{gathered}
$$

where summation is carried out along all isolated singular points (poles) $p=p_{k}$.

The first term [Eq. (12)] of Eq. (11) describes the drift of the equilibrium position of the $n$-th oscillator and is governed by the relaxation-retardation processes occurring in the mechanical system, while the second term [Eq. (13)] describes its damped vibrations around the drifting position of equilibrium and is determined by the inertia forces and dissipative forces.

For utilizing Eqs. (11) to (13) to construct the final expression for $Y_{n}(t)$, the root $p_{n}=-r_{n} e^{ \pm i \psi}=\alpha_{n} \pm i \Omega_{n}$ locus of the characteristic Eq. (10) needs to be investigated, where $\alpha_{n}$ and $\Omega_{n}$ are the damping coefficient and the vibration frequency of the $n$-th oscillator, respectively. This procedure is described in detail in refs. [1, 9].

Knowing the roots of the characteristic equation, it is possible to construct the Green function $G_{n}(t)$ on the basis of Eqs. (11) to (13) if $\bar{F}_{n}=1$ in Eq. (7) [6]

$$
\begin{gathered}
G_{n}(t)=A_{0 n}(t)+A_{n} e^{-\alpha_{n} t} \sin \left(\Omega_{n} t-\varphi_{n}\right), \\
A_{n}=2\left[4 r_{n}^{2}+\gamma^{2} æ_{n}^{2} r_{n}^{2(\gamma-1)}+4 \gamma æ_{n} r_{n}^{\gamma} \cos (2-\gamma) \psi\right]^{-1 / 2}, \\
\tan \varphi_{n}=-\frac{2 r_{n} \cos \psi+\gamma æ_{n} r_{n}^{\gamma-1} \cos (1-\gamma) \psi}{2 r_{n} \sin \psi-\gamma æ_{n} r_{n}^{\gamma-1} \sin (1-\gamma) \psi}, \\
A_{0 n}(t)=\int_{0}^{\infty} r^{-1} B_{n}(\tau) \exp (-t / \tau) \mathrm{d} \tau,
\end{gathered}
$$

$B_{n}(\tau)=\frac{\sin \pi \gamma}{\pi} \frac{\tau\left(1+\tau^{2} \omega_{n}^{2}\right)^{-1}}{\left(1+\tau^{2} \omega_{n}^{2}\right) æ_{n}^{-1} \tau_{n}^{\gamma-2}+\left(1+\tau^{2} \omega_{n}^{2}\right)^{-1} æ_{n} \tau_{n}^{2-\gamma}+2 \cos \pi \gamma}$

is the function of the distribution of the retardation times corresponding to the $n$-th oscillator, and $æ_{n}^{2}=\omega_{n}^{2} \tau_{n}^{\gamma}$. Knowing the Green function, $Y_{n}(t)$ is found as

$$
Y_{n}(t)=\int_{0}^{t} G_{n}\left(t-t^{\prime}\right) F_{n}\left(t^{\prime}\right) \mathrm{d} t^{\prime} .
$$


Below, it will be shown how an infinite set of uncoupled equations in the generalized displacements could be found for thin plates and cylindrical shells, i.e., for systems with infinite degrees of freedom.

\section{Free vibrations of an elastic plate in a viscous medium}

Rossikhin and Shitikova [11] were among the first to investigate the dynamic response of an elastic rectangular plate vibrating in a viscous medium, the damping features of which are modeled by fractional derivatives. The equations describing in-plane and transverse vibrations of such a plate in the dimensionless form are written as

$$
\begin{gathered}
\frac{\partial^{2} u_{1}}{\partial x^{2}}+\frac{1-v}{2} \beta_{1}^{2} \frac{\partial^{2} u_{1}}{\partial y^{2}}+\frac{1+v}{2} \beta_{1} \frac{\partial^{2} u_{2}}{\partial x \partial y}=\ddot{u}_{1}+\mu D^{\gamma} u_{1}-q_{1} \\
\beta_{1}^{2} \frac{\partial^{2} u_{2}}{\partial y^{2}}+\frac{1-v}{2} \frac{\partial^{2} u_{2}}{\partial x^{2}}+\frac{1+v}{2} \beta_{1} \frac{\partial^{2} u_{1}}{\partial x \partial y}=\ddot{u}_{2}+\mu D^{\gamma} u_{2}-q_{2} \\
\frac{\beta_{2}^{2}}{12}\left(\frac{\partial^{4} u_{3}}{\partial x^{4}}+2 \beta_{1}^{2} \frac{\partial^{4} u_{3}}{\partial x^{2} \partial y^{2}}+\beta_{1}^{4} \frac{\partial^{4} u_{3}}{\partial y^{4}}\right)=-\ddot{u}_{3}-\mu D^{\gamma} u_{3}+q_{3},
\end{gathered}
$$

where $u_{1}(x, y, t), u_{2}(x, y, t)$, and $u_{3}(x, y, t)$ are displacements of the points of the plate's median surface in three mutually orthogonal directions $x, y, z$, two of which, $x$ and $y$, lie in the plate surface, and the third one, $z$, is out of the plate plane; $q_{1}, q_{2}$, and $q_{3}$ are the intensities of the given external loads applied in the $x$-, $y$-, and $z$-directions, respectively; $v$ is Poisson's ratio; $\mu$ is the damping coefficient; $\beta_{1}=a / b$ and $\beta_{2}=h / a$ are the parameters depending on the plate's dimensions; $a$ and $b$ are the plate's dimensions along the $x$ - and $y$-axis, respectively; $h$ is the plate thickness; and the fractional derivative is defined as [1]

$$
D^{\gamma} Z=\frac{\mathrm{d}}{\mathrm{d} t} \int_{0}^{t} \frac{Z(\tau) \mathrm{d} \tau}{\Gamma(1-\gamma)(t-\tau)^{\gamma}}
$$

With reference to Eqs. (20) to (22), it follows that the inplane and out-of-plane vibrations of such a plate are uncoupled, while the in-plane motions are described by a set of two linear coupled equations [Eqs. (20) and (21)]. That is why the behavior of each mode of the coupled equations was modeled in ref. [11] by the behavior of a two-mass oscillator [12]; in so doing, the oscillators corresponding to different modes are separated from each other.

Below, we will use another approach resulting in the decoupling of the equations for the in-plane vibrations and utilizing equations of one-mass oscillators. Thus, assume for simplicity that Eqs. (20) to (22) are subjected to the boundary conditions of the Navier type. Then, the displacements and the external loads can be represented in the form

$$
\begin{aligned}
& u_{i}(x, y, t)=\sum_{m=1}^{\infty} \sum_{n=1}^{\infty} x_{i m n}(t) \eta_{i m n}(x, y), \quad(i=1,2,3) \\
& q_{i}(x, y, t)=\sum_{m=1}^{\infty} \sum_{n=1}^{\infty} y_{i m n}(t) \eta_{i m n}(x, y), \quad(i=1,2,3),
\end{aligned}
$$

where $m$ and $n$ are integers; $x_{i m n}$ and $y_{i m n}$ are the generalized displacements and forces, respectively; and $\eta_{i m n}(x, y)$ are the eigenfunctions of the given problem:

$$
\begin{aligned}
& \eta_{1 m n}(x, y)=\cos \pi m x \sin \pi n y, \\
& \eta_{2 m n}(x, y)=\sin \pi m x \cos \pi n y, \\
& \eta_{3 m n}(x, y)=\sin \pi m x \sin \pi n y
\end{aligned}
$$

at $0 \leq x \leq 1$ and $0 \leq y \leq 1$.

Substituting Eqs. (23) and (24) in Eqs. (20) to (22), using the conditions of orthogonality of the eigenfunctions (25) on the segments $0 \leq x, y \leq 1$, and applying the Laplace transform to the resulting set of equations, we find

$$
\begin{gathered}
p^{2} \bar{x}_{\alpha m n}+S_{\alpha \beta}^{m n} \bar{x}_{\beta m n}+p^{\gamma} \mu_{\alpha \beta}^{m n} \bar{x}_{\beta m n}=\bar{y}_{\alpha m n}, \\
\left(p^{2}+\mu_{m n} p^{\gamma}+P_{m n}\right) \bar{x}_{3 m n}=\bar{y}_{3 m n},
\end{gathered}
$$

where the Greek letters take on the magnitudes of 1 and 2, the summation is carried out over two repeated indices, the elements of the matrix $S_{\alpha \beta}^{m n}$ and $P_{m n}$ are defined as follows:

$$
\begin{array}{ll}
S_{11}^{m n}=\pi^{2}\left(m^{2}+\frac{1-v}{2} \beta_{1}^{2} n^{2}\right), & S_{12}^{m n}=S_{21}^{m n}=\pi^{2} \frac{1+v}{2} \beta_{1} m n, \\
S_{22}^{m n}=\pi^{2}\left(\frac{1-v}{2} m^{2}+\beta_{1}^{2} n^{2}\right), & P_{m n}=\pi^{4} \frac{\beta_{2}^{2}}{12}\left(m^{2}+\beta_{1}^{2} n^{2}\right)^{2},
\end{array}
$$

and the modal viscosity $\mu_{m n}$ is assumed to be satisfied to the Rayleigh hypothesis, i.e., $\mu_{m n}=æ_{m n} S_{\alpha \beta}^{m n}$ with the value æ taken for convenience as $æ_{m n}=\tau_{m n}^{\gamma}$.

Then, Eq. (26) can be rewritten as

$$
p^{2} \bar{x}_{\alpha m n}+S_{\alpha \beta}^{m n} \bar{x}_{\beta m n}\left(1+p^{\gamma} \tau_{m n}^{\gamma}\right)=\bar{y}_{\alpha m n} .
$$

As the matrix $S_{\alpha \beta}^{m n}$ is symmetric, then it possesses two real eigenvalues

$$
p_{i}^{2}=-\omega_{i m n}^{2}, \quad \omega_{1 m n}^{2}=\pi^{2}\left(m^{2}+\beta_{1}^{2} n^{2}\right), \quad \omega_{2 m n}^{2}=\pi^{2} \frac{1-v}{2}\left(m^{2}+\beta_{1}^{2} n^{2}\right),
$$


which are in correspondence with two mutually orthogonal eigenvectors

$$
\begin{aligned}
& l_{m n}^{\mathrm{I}}\left\{l_{1 m n}^{\mathrm{I}}=\frac{\pi m}{\omega_{1 m n}}, \quad l_{2 m n}^{\mathrm{I}}=\frac{\pi \beta_{1} n}{\omega_{1 m n}}\right\} \\
& l_{m n}^{\mathrm{II}}\left\{l_{l^{\mathrm{I}} \mathrm{I}}^{\mathrm{I}}=\frac{\pi \beta_{1} n}{\omega_{1 m n}}, \quad l_{2 m n}^{\mathrm{II}}=-\frac{\pi m}{\omega_{1 m n}}\right\}
\end{aligned}
$$

Thus, the matrix $S_{\alpha \beta}^{m n}$ and the vectors $\bar{x}_{\alpha m n}$ and $\bar{y}_{\alpha m n}$ can be expanded in terms of the vectors in Eqs. (30) and (31) as

$$
\begin{aligned}
& S_{\alpha \beta}^{m n}=\omega_{1 m n}^{2} l_{\alpha m n}^{\mathrm{I}} l_{\beta m n}^{\mathrm{I}}+\omega_{2 m n}^{2} l_{\alpha m n}^{\mathrm{II}} l_{\beta m n}^{\mathrm{II}} \\
& \bar{X}_{\alpha m n}=\bar{X}_{1 m n} l_{\alpha m n}^{\mathrm{I}}+\bar{X}_{2 m n} l_{\alpha m n}^{\mathrm{II}} \\
& \bar{y}_{\alpha m n}=\bar{Y}_{1 m n} l_{\alpha m n}^{\mathrm{I}}+\bar{Y}_{2 m n} l_{\alpha m n}^{\mathrm{II}}
\end{aligned}
$$

Substituting (32) in Eq. (28), and multiplying the net relationships successively by $l_{\alpha m n}^{\mathrm{I}}$ and $l_{\alpha m n}^{\mathrm{II}}$, we obtain two equations

$$
\begin{aligned}
& {\left[p^{2}+\omega_{1 m n}^{2}\left(1+p^{\gamma} \tau_{m n}^{\gamma}\right)\right] \bar{X}_{1 m n}=\bar{Y}_{1 m n},} \\
& {\left[p^{2}+\omega_{2 m n}^{2}\left(1+p^{\gamma} \tau_{m n}^{\gamma}\right)\right] \bar{X}_{2 m n}=\bar{Y}_{2 m n} .}
\end{aligned}
$$

Eq. (27) rewritten in the similar form

$$
\left[p^{2}+\Omega_{m n}^{2}\left(1+p^{\gamma} \tau_{1 m n}\right)\right] \bar{X}_{3 m n}=\bar{Y}_{3 m n}
$$

should beadded to Eqs. (34) and (34), where $\Omega_{m n}^{2}=P_{m n}$ is the natural frequency of the out-of-plane vertical vibrations.

Reference to Eqs. (33) to (35) shows that these equations differ from Eq. (6) only by the notations of the values entering in these equations, and thus, further procedure for the construction of the solution should be the same as for the Kelvin-Voigt fractional one-mass oscillator (see details in Section 2 and in refs. $[1,9])$.

\section{Linear vibrations of a circular cylindrical shell being in a viscous medium}

Now let us consider an elastic circular cylindrical shell of radius $R$ and length $l$ being in a viscous medium. Equations describing vibrations of such a shell in the dimensionless form can be written as

$\frac{\partial^{2} u_{1}}{\partial x^{2}}+\frac{1-v}{2} \beta_{1}^{2} \frac{\partial^{2} u_{1}}{\partial \varphi^{2}}+\frac{1+v}{2} \beta_{1} \frac{\partial^{2} u_{2}}{\partial \chi \partial \varphi}-v \beta_{1} \frac{\partial u_{3}}{\partial x}=\ddot{u}_{1}+\mu_{11} D^{\gamma} u_{1}-q_{1}$,

$$
\begin{aligned}
& \beta_{1}^{2} \frac{\partial^{2} u_{2}}{\partial \varphi^{2}}+\frac{1-v}{2} \frac{\partial^{2} u_{2}}{\partial x^{2}}+\frac{1+v}{2} \beta_{1} \frac{\partial^{2} u_{1}}{\partial x \partial \varphi}-\beta_{1}^{2} \frac{\partial u_{3}}{\partial \varphi}=\ddot{u}_{2}+\mu_{22} D^{\gamma} u_{2}-q_{2}, \\
& \frac{\beta_{2}^{2}}{12}\left(\frac{\partial^{4} u_{3}}{\partial x^{4}}+2 \beta_{1}^{2} \frac{\partial^{4} u_{3}}{\partial x^{2} \partial \varphi^{2}}+\beta_{1}^{4} \frac{\partial^{4} u_{3}}{\partial \varphi^{4}}\right)+\beta_{1}^{2} u_{3}-v \beta_{1} \frac{\partial u_{1}}{\partial x}-\beta_{1}^{2} \frac{\partial u_{2}}{\partial \varphi} \\
& \quad=-\ddot{u}_{3}-\mu_{33} D^{\gamma} u_{3}+q_{3},
\end{aligned}
$$

where the $x$-axis is directed along the axis of the cylinder; $\varphi$ is the polar angle in the plane perpendicular to the $x$-axis; $u_{1}(x, \varphi, t), u_{2}(x, \varphi, t)$, and $u_{3}(x, \varphi, t)$ are the dimensionless displacements of the points of the shell's median surface in three mutually orthogonal directions $\chi, \varphi, r, r$ is the polar radius; $q_{1}, q_{2}$, and $q_{3}$ are the intensities of the given external loads applied in the $x-, \varphi$-, and $r$-directions, respectively; $v$ is Poisson's ratio; $\mu_{i i}$ are the damping coefficients; $\beta_{1}=l / R$ and $\beta_{2}=h / l$ are the parameters depending on the shell's dimensions; and $h$ is the shell thickness.

Free damped transverse vibrations of a circular cylindrical shell described by Eq. (38) with $u_{1}=u_{2}=0$ have been considered in ref. [13]. The set of Eqs. (36) to (38) also admits the solution of the Navier-type Eqs. (23) and (24), where the variable $y$ should be substituted by $\varphi$.

Substituting then Eqs. (23) and (24) in Eqs. (36) to (38), using the conditions of orthogonality of the eigenfunctions within the domains of $0 \leq x \leq 1$ and $0 \leq \varphi \leq 2 \pi$, and applying the Laplace transformation to the resulting equations, we obtain

$$
\left[p^{2} \delta_{i j}+\mu_{i j}^{m n} p^{\gamma}+S_{i j}^{m n}\right] \overrightarrow{\bar{X}}_{j m n}=\overrightarrow{\bar{q}}_{i m n},
$$

where the Latin indices take on the magnitudes of 1,2 , and 3; the summation is carried out over two repeated indices, $\mu_{i j}^{m n}=\tau^{\gamma} S_{i j}^{m n}$; and the elements of the matrix $S_{i j}^{m n}$ are defined as follows:

$$
\begin{aligned}
& S_{11}^{m n}=\pi^{2}\left(m^{2}+\frac{1-v}{2} \beta_{1}^{2} n^{2}\right), \quad S_{12}^{m n}=S_{21}^{m n}=\pi^{2} \frac{1+v}{2} \beta_{1} m n, \\
& S_{13}^{m n}=S_{31}^{m n}=v \beta_{1} \pi m, \quad S_{23}^{m n}=S_{32}^{m n}=\beta_{1}^{2} \pi n, \\
& S_{22}^{m n}=\pi^{2}\left(\frac{1-v}{2} m^{2}+\beta_{1}^{2} n^{2}\right), \quad S_{33}^{m n}=\pi^{4} \frac{\beta_{2}^{2}}{12}\left(m^{2}+\beta_{1}^{2} n^{2}\right)^{2} .
\end{aligned}
$$

As the matrix $S_{i j}^{m n}$ is symmetric, then it has three real eigenvalues

$$
p_{i}^{2}=-\Omega_{i m n}^{2}, \quad(i=1,2,3)
$$

which are in correspondence with three mutually orthogonal eigenvectors

$$
\vec{L}_{m n}^{\mathrm{I}}\left\{L_{i m n}^{\mathrm{I}}\right\}, \quad \vec{L}_{m n}^{\mathrm{II}}\left\{L_{i m n}^{\mathrm{II}}\right\}, \quad \vec{L}_{m n}^{\mathrm{III}}\left\{L_{i m n}^{\mathrm{III}}\right\} .
$$


Thus, the matrix $S_{i j}^{m n}$ and the vectors $\overrightarrow{\bar{x}}_{i m n}$ and $\overrightarrow{\bar{q}}_{i m n}$ can be expanded in terms of the vectors in Eq. (41) as

$$
\begin{gathered}
S_{i j}^{m n}=\Omega_{1 m n}^{2} L_{i m n}^{\mathrm{I}} L_{j m n}^{\mathrm{I}}+\Omega_{2 m n}^{2} L_{i m n}^{\mathrm{II}} L_{j m n}^{\mathrm{II}}+\Omega_{3 m n}^{2} L_{i m n}^{\mathrm{III}} L_{j m n}^{\mathrm{III}}, \\
\bar{X}_{i m n}=\bar{X}_{1 m n} L_{i m n}^{\mathrm{I}}+\bar{X}_{2 m n} L_{i m n}^{\mathrm{II}}+\bar{X}_{3 m n} L_{i m n}^{\mathrm{III}}, \\
\bar{q}_{i m n}=\bar{Q}_{1 m n} L_{i m n}^{\mathrm{I}}+\bar{Q}_{2 m n} L_{i m n}^{\mathrm{II}}+\bar{Q}_{3 m n} L_{i m n}^{\mathrm{III}} .
\end{gathered}
$$

Substituting Eqs. (42) to (44) in Eq. (39) yields

$$
\begin{aligned}
& {\left[p^{2} \delta_{i j}+\left(1+p^{\gamma} \tau^{\gamma}\right)\left(\Omega_{1 m n}^{2} L_{i m n}^{\mathrm{I}} L_{j m n}^{\mathrm{I}}+\Omega_{2 m n}^{2} L_{i m n}^{\mathrm{II}} L_{j m n}^{\mathrm{II}}+\Omega_{3 m n}^{2} L_{i m n}^{\mathrm{III}} L_{j m n}^{\mathrm{III}}\right)\right]} \\
& \quad \times\left(\bar{X}_{1 m n} L_{j m n}^{\mathrm{I}}+\bar{X}_{2 m n} L_{j m n}^{\mathrm{II}}+\bar{X}_{3 m n} L_{j m n}^{\mathrm{III}}\right) \\
& \quad=\bar{Q}_{1 m n} L_{i m n}^{\mathrm{I}}+\bar{Q}_{2 m n} L_{i m n}^{\mathrm{II}}+\bar{Q}_{3 m n} L_{i m n}^{\mathrm{III}} .
\end{aligned}
$$

Multiplying (45) successively by $L_{i m n}^{\mathrm{I}}, L_{i m n}^{\mathrm{II}}$, and $L_{i m n}^{\mathrm{III}}$, and considering that

$$
\begin{aligned}
& L_{i m n}^{\mathrm{I}} L_{i m n}^{\mathrm{II}}=L_{i m n}^{\mathrm{I}} L_{i m n}^{\mathrm{III}}=L_{i m n}^{\mathrm{II}} L_{i m n}^{\mathrm{III}}=0, \\
& L_{i m n}^{\mathrm{I}} L_{i m n}^{\mathrm{I}}=L_{i m n}^{\mathrm{II}} L_{i m n}^{\mathrm{II}}=L_{i m n}^{\mathrm{III}} L_{i m n}^{\mathrm{III}}=1,
\end{aligned}
$$

we obtain three equations

$$
\begin{aligned}
& {\left[p^{2}+\Omega_{1 m n}^{2}\left(1+p^{\gamma} \tau_{m n}^{\gamma}\right)\right] \bar{X}_{1 m n}=\bar{Q}_{1 m n},} \\
& {\left[p^{2}+\Omega_{2 m n}^{2}\left(1+p^{\gamma} \tau_{m n}^{\gamma}\right)\right] \bar{X}_{2 m n}=\bar{Q}_{2 m n},}
\end{aligned}
$$

$$
\left[p^{2}+\Omega_{3 m n}^{2}\left(1+p^{\gamma} \tau_{m n}^{\gamma}\right)\right] \bar{X}_{3 m n}=\bar{Q}_{3 m n} .
$$

Reference to Eqs. (47)-(49) shows that these equations differ from Eq. (6) only by notations of the values entering in these equations, and thus, further procedure for the construction of the solution should be the same as that for mechanical oscillators.

\section{Conclusion}

It is shown that the introduction of the modal viscosity (i.e., the assumption that each mode of vibration has its own damping coefficient and its own retardation time) together with the Rayleigh hypothesis allows one to reduce the problem of linear vibrations of thin elastic bodies, such as a rectangular plate and a circular cylindrical shell, in a viscoelastic medium to the problem of vibrations of an infinite set of viscoelastic oscillators based on the fractional derivative Kelvin-Voigt model.

The suggested approach for decoupling equations of motion could be generalized for solving dynamic problems of non-linear weakly damped vibrations of thin bodies [14].

Acknowledgments: The research work reported here was made possible by the joint grant from the Russian Foundation for Basic Research No. 10-01-92004HHC-a and the National Science Council of Taiwan No. RP10E02.

\section{References}

[1] Rossikhin YuA, Shitikova MV. Appl. Mech. Rev. 1997, 50, $15-67$.

[2] Gaul L. Mech. Syst. Signal. Process. 1999, 13, 1-30.

[3] Shimizu N, Zhang W. JSME Int. J. Ser. C 1999, 42, 825-837.

[4] French M, Rogers J. In IMAC-IX: A Conference on Structural Dynamics. Kissimmee, FL, Ed. USA, 5-8 Feb 2001, vol. 1, 2001, pp. 305-309.

[5] Xu M, Tan W. Sci. China Ser. G Phys. Mech. Astron. 2006, 49, 257-272.

[6] Rossikhin YuA, Shitikova MV. Appl. Mech. Rev. 2010, 63, 010801-1-52.

[7] Rossikhin YuA. Appl. Mech. Rev. 2010, 63, 010701-1-12.

[8] Clough RW, Penzien J. Dynamics of Structures. McGraw-Hill: New York, 1975.

[9] Rossikhin YuA, Shitikova MV. Acta Mech. 1997, 120, 109-125.
[10] Abdel-Ghaffar AM, Scanlan RH. ASCE J. Eng. Mech. 1985, 111, 463-482.

[11] Rossikhin YuA, Shitikova MV. Signal Process. 2006, 86, 2703-2711.

[12] Rossikhin YuA, Shitikova MV. Int. J. Eng. Sci. 2001, 39, 149-176.

[13] Rossikhin YuA, Shitikova MV, Ovsjannikova El. In Proceeding 5th International. Conference on Vibration Problems ICOVP-2001. Moscow, 8-10 October 2001, IMASH: Moscow, 2002, pp. 378-381.

[14] Rossikhin YuA, Shitikova MV. In Abstract Book of the 23rd International Congress of Theoretical and Applied Mechanics ICTAM 2012. Beijing, China, August 19-24, 2012, China Science Literature Publishing House, 2012, pp. 6-7. 